\title{
The Role of Cyclooxygenase- I and -2 in Sevoflurane-Induced Postconditioning Against Myocardial Infarction
}

Seminars in Cardiothoracic and

Vascular Anesthesia

2014, Vol. 18(3) 272-280

(C) The Author(s) 2014

Reprints and permissions:

sagepub.com/journalsPermissions.nav

DOI: $10.1177 / 10892532 \mid 4523683$

scv.sagepub.com

(SAGE

\author{
Jan Stumpner, MD', Tobias Tischer-Zeitz, MD', Anja Frank, MD', Christopher \\ Lotz, MD', Andreas Redel, MD², Markus Lange, MD PhD ${ }^{3}$, Franz Kehl, MD PhD ${ }^{4}$, \\ Norbert Roewer, MD PhD', and Thorsten Smul, MD'
}

\begin{abstract}
Cyclooxygenase (COX)-2 mediates ischemic pre- and postconditioning as well as anesthetic-induced preconditioning. However, the role of COX-I and -2 in anesthetic-induced postconditioning has not been investigated. We evaluated the role of COX-I and -2 in sevoflurane-induced postconditioning in vivo. Pentobarbital-anaesthetized male C57BL/6 mice were subjected to 45 minutes of coronary artery occlusion and 3 hours of reperfusion. Animals received either no intervention, the vehicle dimethyl sulfoxide (DMSO, $10 \mu \mathrm{L} / \mathrm{g}$ intraperitoneally), acetylsalicylic acid (ASA, $5 \mu \mathrm{g} / \mathrm{g}$ intraperitoneally), the selective COX-I inhibitor SC-560 ( $10 \mu \mathrm{g} / \mathrm{g}$ intraperitoneally), or the selective COX-2 inhibitor NS398 (5 $\mathrm{\mu g} / \mathrm{g}$ intraperitoneally). I.0 MAC (minimum alveolar concentration) sevoflurane was administered for 18 minutes during early reperfusion either alone or in combination with ASA, SC-560, and NS-398. Infarct size was determined with triphenyltetrazolium chloride. Statistical analysis was performed using I-way and 2-way analyses of variance with post hoc Duncan testing. The infarct size in the control group was $44 \% \pm 9 \%$. DMSO (42\% $\pm 7 \%)$, ASA (36\% $\pm 6 \%)$, and NS-398 $(44 \% \pm 18 \%)$ had no effect on infarct size. Sevoflurane $(17 \% \pm 4 \% ; P<.05)$ and SC-560 $(26 \% \pm 10 \% ; P<.05)$ significantly reduced the infarct size compared with control condition. Sevoflurane-induced postconditioning was not abolished by ASA $(16 \% \pm 5 \%)$ and SC-560 (22\% $\pm 4 \%)$. NS-398 abolished sevoflurane-induced postconditioning $(33 \% \pm$ 14\%). It was concluded that sevoflurane induces postconditioning in mice. Inhibition of COX-I elicits a myocardial infarct size reduction and does not abolish sevoflurane-induced postconditioning. Blockade of COX-2 abolishes sevofluraneinduced postconditioning. These results indicate that sevoflurane-induced postconditioning is mediated by COX-2.
\end{abstract}

\section{Keywords}

cyclooxygenase-I, cardiac anesthesia, ischemia-reperfusion injury, volatile anesthetics, postconditioning, cyclooxygenase-2

\section{Introduction}

Ischemic heart disease is the leading cause of death in developed nations. Extending myocardial ischemic tolerance could be a promising strategy to improve patient outcome after acute cardiovascular events. Volatile anesthetics such as isoflurane, desflurane, and sevoflurane confer cardioprotection by inducing preconditioning ${ }^{1-3}$ and postconditioning. ${ }^{4-6}$ Postconditioning might be of exceptional relevance in the clinical setting since myocardial ischemia usually is unpredictable, very much limiting the use of preconditioning. Improved outcome via pharmaceutical postconditioning is indisputable in animal studies but difficult to detect in patients. ${ }^{7}$ Various mechanisms, such as age, comorbidities, and the accompanying pharmacotherapy, can interfere with cardioprotection, thus presenting a challenge for successful clinical translation.

Inhibitors of cyclooxygenase (COX)-1 and -2 are widely prescribed because of their anti-inflammatory and analgesic properties. However, application of COX-2 inhibitors is critical in patients with a high cardiovascular risk profile. ${ }^{8-10}$ In particular, administration of valdecoxib and its oral prodrug parecoxib was associated with serious cardiovascular events after coronary artery bypass graft

\footnotetext{
'Department of Anaesthesia and Critical Care, University of Wuerzburg, Wuerzburg, Germany

${ }^{2}$ Department of Anesthesia, University of Regensburg, Regensburg, Germany

${ }^{3}$ Department of Anesthesia and Critical Care Medicine, Mathias-Spital, Rheine, Germany

${ }^{4}$ Department of Anesthesiology and Critical Care, Hospital of Karlsruhe, Karlsruhe, Germany
}

\section{Corresponding Author:}

Jan Stumpner, University of Wuerzburg, Department of Anaesthesia and Critical Care, Oberduerrbacher Str. 6, Wuerzburg, 97080,

Germany.

Email: stumpner_j@ukw.de 
surgery. ${ }^{10}$ Therefore, some of the COX-2 inhibitors were taken off the market or were not approved in several countries. In the experimental setting, COX-2 inhibitors abolish the acute phase of ischemic and volatile anesthetic-induced preconditioning against myocardial infarction, indicating an important role for COX-2 in cardioprotection. ${ }^{1}$

Whether cyclooxygenase enzymes 1 and 2 play a role in the signal transduction cascade of anesthetic-induced postconditioning has not been investigated to date. Hence, we tested the hypothesis that blockade of COX-1 and -2 abolishes sevoflurane-induced postconditioning in the murine heart in vivo.

\section{Methods}

\section{Ethical Approval}

All experimental procedures used in this investigation were reviewed and approved by the Animal Care and Use Committee of the Government of Lower Franconia, Bavaria, Germany. All experiments were in accordance with the Guide for the Care and Use of Laboratory Animals ${ }^{11}$ and conformed to the Guiding Principles in the Care and Use of Animals of the American Physiological Society.

\section{Animals}

Male C57BL/6 mice (8-12 weeks old) were purchased from Harlan (Horst, The Netherlands). Animals were housed under controlled conditions $\left(22^{\circ} \mathrm{C}, 55 \%-65 \%\right.$ humidity, 12-hour light-dark cycle) and were allowed free access to water and a standard laboratory chow.

\section{Instrumentation and Surgical Procedures}

Instrumentation and surgical procedures were performed as described previously. ${ }^{12,13}$ Briefly, mice were anesthetized with an intraperitoneal injection of $60 \mu \mathrm{g} / \mathrm{g}$ sodium pentobarbital (Merial, Hallbergmoos, Germany), and repeated intraperitoneal injections were given as needed to maintain anesthesia. Rectal temperature was maintained at $37.0^{\circ} \mathrm{C} \pm 0.1^{\circ} \mathrm{C}$ using a servo-controlled heating pad (FMI, Seeheim, Germany). After intubation of the trachea, mice were ventilated with a 50\%/50\% air-oxygen mixture using a small rodent ventilator (SAR-P 830, CWE Inc, Ardmore, Pennsylvania) operating in pressure-controlled mode. A 3-lead needle-probe electrocardiogram (ECG) was attached to continuously monitor heart rate and ST-segment elevation. Saline-filled polyethylene catheters were placed into the right common carotid artery for measurement of mean arterial blood pressure and into the right jugular vein for continuous fluid administration $(20 \mu \mathrm{L} / \mathrm{g} / \mathrm{h})$. A left thoracotomy at the fourth intercostal space was performed, and the left anterior descending coronary artery (LAD) was exposed. The ligature was set as previously described. ${ }^{12}$ Coronary artery occlusion was achieved using the hanging weight system $^{14}$ and was verified by ECG ST-segment elevation and paleness of the myocardial area at risk (AAR). Adequate reperfusion was verified by epicardial hyperemia and reversion of ECG changes.

\section{Experimental Protocol}

After completion of surgical procedures, investigators randomly assigned mice to 1 of the 9 study groups by opening a sealed envelope containing information about the study group. Group size was $\mathrm{n}=7$ in each group. The experimental protocol is illustrated in Figure 1.

All mice were allowed a 30-minute equilibration period. Myocardial ischemia was induced by 45 minutes of coronary artery occlusion (CAO) followed by 3 hours of reperfusion. Control animals (CON) received no treatment before CAO. In group 2 (DMSO), the vehicle dimethyl sulfoxide (DMSO, $10 \mu \mathrm{L} / \mathrm{g}$ ) was injected intraperitoneally (i.p.) 10 minutes prior to the end of CAO. In group 3 (SEVO), 1.0 MAC (minimum alveolar concentration, 3,4 Vol- $\%{ }^{15}$ ) sevoflurane was given for 18 minutes starting 3 minutes prior to the end of CAO. Sevoflurane concentration was gradually increased from 0.0 to $1.0 \mathrm{MAC}$ and gradually decreased from $1.0 \mathrm{MAC}$ to $0.0 \mathrm{MAC}$ over a time period of 2 minutes. Ten minutes prior to the end of $\mathrm{CAO}$, animals received the nonselective COX inhibitor acetylsalicylic acid (ASA, $5 \mu \mathrm{g} / \mathrm{g}$ ) either alone (ASA) or in combination with sevoflurane (SEVO+ASA). To evaluate the role of COX-1, the selective COX-1 inhibitor SC-560 $\left(10 \mu \mathrm{g} / \mathrm{g}^{16}\right)$ was given i.p. 10 minutes prior to the end of $\mathrm{CAO}$, either alone (SC-560) or in combination with sevoflurane (SEVO+SC-560). The selective COX-2 inhibitor NS-398 $\left(5 \mu \mathrm{g} / \mathrm{g}^{16}\right)$ was administered i.p. either alone (NS398) or in combination with sevoflurane (SEVO+NS-398) to investigate the role of COX-2 in sevoflurane-induced postconditioning.

\section{Measurement of Myocardial Infarct Size}

Myocardial infarct size (IS) and area at risk (AAR) were determined using methods previously described. ${ }^{12}$ Briefly, after 3 hours of reperfusion, the LAD was reoccluded and $1 \mathrm{~mL}$ of Evans Blue $(0.1 \mathrm{~g} / \mathrm{mL}$, Sigma-Aldrich, Taufkirchen, Germany) was slowly injected into the carotid artery. After intraperitoneal injection of a lethal dose of sodium pentobarbital $(150 \mu \mathrm{g} / \mathrm{g})$, the heart was rapidly excised. The left ventricle was separated and cut into 7 or 8 transversal slices of each $1 \mathrm{~mm}$ thickness. Slices were incubated in 2,3,5-triphenyltetrazolium chloride (20 $\mathrm{mg} / \mathrm{mL}$ ) for 30 minutes at $37^{\circ} \mathrm{C}$. After overnight fixation in $10 \%$ formaldehyde, slices were weighted and digitally 


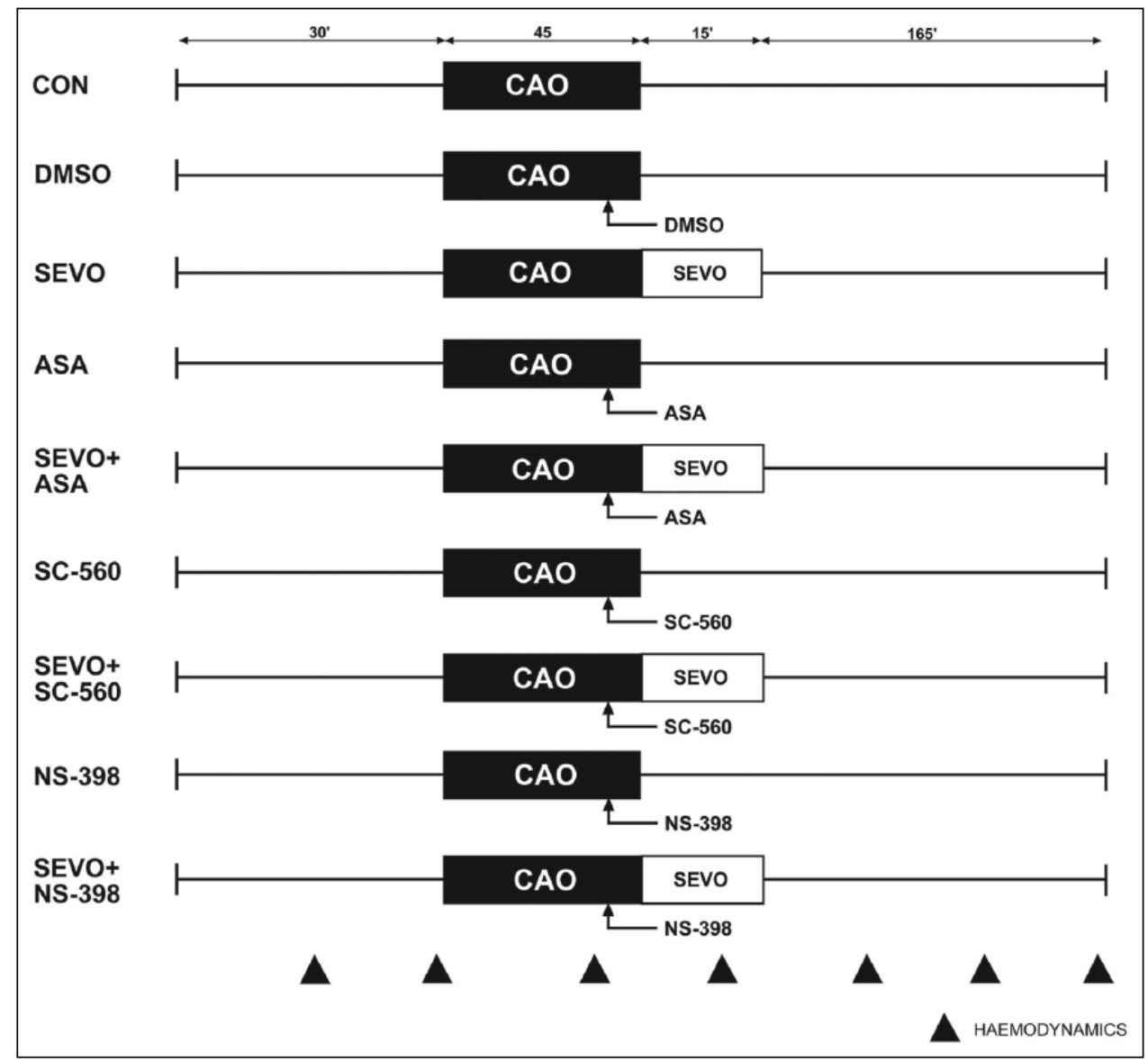

Figure I. Schematic diagram illustrating the experimental protocol. CAO is coronary artery occlusion. CON, control group. DMSO, dimethyl sulfoxide $(10 \mu \mathrm{L} / \mathrm{g})$ intraperitoneally (i.p.) 10 minutes prior to the onset of reperfusion. SEVO, I.O MAC (minimum alveolar concentration) sevoflurane for 18 minutes starting 3 minutes prior to the onset of reperfusion. ASA, acetylsalicylic acid ( 5 $\mu \mathrm{g} / \mathrm{g})$ i.p. 10 minutes prior to the onset of reperfusion. SEVO+ASA, acetylsalicylic acid $(5 \mu \mathrm{g} / \mathrm{g})$ i.p. 7 minutes prior to sevoflurane (I.0 MAC). SC-560, SC-560 (I0 $\mu \mathrm{g} / \mathrm{g})$ i.p. 10 minutes prior to the onset of reperfusion. SEVO+SC-560, SC-560 (I0 $\mu \mathrm{g} / \mathrm{g})$ i.p. 7 minutes prior to sevoflurane (I.0 MAC). NS-398, NS-398 (5 $\mathrm{gg} / \mathrm{g})$ i.p. 10 minutes prior to the onset of reperfusion. SEVO+NS-398, NS-398 $(5 \mu \mathrm{g} / \mathrm{g})$ i.p. 7 minutes prior to sevoflurane (I.0 MAC).

photographed. Photographs were analyzed using AdobePhotoshop CS 8.0.1 (Adobe Systems Inc, San Jose, California), and the normal zone, AAR, and IS were determined gravitoplanimetrically by a blinded investigator. Animals with an AAR of less than $20 \%$ were excluded from the study.

\section{Data Acquisition and Statistical Analysis}

ECG, systemic hemodynamic parameters, and body temperature were continuously recorded and analyzed on a personal computer (Fujitsu Siemens, Augsburg, Germany) using a hemodynamic data acquisition and analysis software (Notocord hem 3.5, Croissy sur Seine, France).

Drawing from other studies on the same experimental model, we expected a myocardial IS between $45 \%$ and $50 \%$ (IS/AAR). Power analysis revealed a group size of $\mathrm{n}=7$ to detect a difference in means of $20 \%$ with a power of 0.8 at a $\alpha$-level of 0.05 . Statistical analyses were done by analyses of variance (ANOVAs), which were based on 2 -tailed $F$ tests for comparison of components of the factors' total deviation. Analysis for body weight, left ventricle weight, left ventricle weight/body weight, AAR, IS, IS/ left ventricle weight, and AAR/left ventricle weight was performed using 1-way ANOVA including the factor treatment (CON vs DMSO vs SEVO vs ASA vs SEVO+ASA vs SC-560 vs SEVO+SC-560 vs NS-398 vs SEVO+NS-398) and post hoc Duncan's test for significant main effects and interactions. Analysis of hemodynamic data was performed by a $9 \times 7$ ANOVA for repeated measures, including the between-factor treatment (CON vs DMSO vs SEVO vs ASA vs SEVO+ASA vs SC-560 vs SEVO+SC-560 vs NS-398 vs SEVO+NS-398) and the within-factor time point (baseline vs pre-CAO vs CAO vs 
Table I. Systemic Hemodynamic Parameters. ${ }^{a}$

\begin{tabular}{|c|c|c|c|c|c|c|c|}
\hline & \multirow[b]{2}{*}{$\mathrm{BL}$} & \multirow[b]{2}{*}{ Pre-CAO } & \multirow[b]{2}{*}{ CAO } & \multirow[b]{2}{*}{ Post-CAO } & \multicolumn{3}{|c|}{ Reperfusion } \\
\hline & & & & & $60 \mathrm{~min}$ & $120 \mathrm{~min}$ & $180 \mathrm{~min}$ \\
\hline \multicolumn{8}{|l|}{ Heart rate, $\min ^{-1}$} \\
\hline $\mathrm{CON}$ & $459 \pm 55$ & $453 \pm 58$ & $450 \pm 59$ & $432 \pm 37$ & $448 \pm 31$ & $435 \pm 30$ & $444 \pm 32$ \\
\hline DMSO & $466 \pm 33$ & $474 \pm 18$ & $459 \pm 35$ & $466 \pm 48$ & $460 \pm 33$ & $458 \pm 19$ & $461 \pm 49$ \\
\hline SEVO & $462 \pm 18$ & $462 \pm 11$ & $452 \pm 34$ & $406 \pm 36^{\mathrm{b}}$ & $433 \pm 31$ & $436 \pm 20$ & $438 \pm 19$ \\
\hline ASA & $468 \pm 32$ & $460 \pm 38$ & $449 \pm 21$ & $457 \pm 35$ & $430 \pm 36$ & $436 \pm 36$ & $454 \pm 31$ \\
\hline SEVO+ASA & $463 \pm 47$ & $46 I \pm 46$ & $44 I \pm 25$ & $40 \mathrm{I} \pm 42^{\mathrm{b}}$ & $435 \pm 46$ & $456 \pm 65$ & $433 \pm 33$ \\
\hline SC-560 & $457 \pm 35$ & $467 \pm 35$ & $468 \pm 34$ & $482 \pm 53$ & $466 \pm 36$ & $468 \pm 46$ & $472 \pm 20$ \\
\hline SEVO+SC-560 & $493 \pm 23$ & $491 \pm 58$ & $470 \pm 46$ & $420 \pm 45$ & $473 \pm 33$ & $470 \pm 22$ & $461 \pm 32$ \\
\hline NS-398 & $461 \pm 32$ & $464 \pm 35$ & $466 \pm 27$ & $467 \pm 58$ & $478 \pm 38$ & $470 \pm 24$ & $475 \pm 43$ \\
\hline SEVO+NS-398 & $471 \pm 12$ & $478 \pm 25$ & $469 \pm 31$ & $433 \pm 41$ & $470 \pm 39$ & $473 \pm 31$ & $472 \pm 39$ \\
\hline \multicolumn{8}{|c|}{ Mean arterial pressure, $\mathrm{mm} \mathrm{Hg}$} \\
\hline CON & $75 \pm 3$ & $74 \pm 7$ & $60 \pm 4$ & $67 \pm 5$ & $67 \pm 8$ & $67 \pm 12$ & $67 \pm 13$ \\
\hline DMSO & $68 \pm 7$ & $64 \pm 6$ & $60 \pm 9^{b}$ & $67 \pm 11$ & $67 \pm 9$ & $68 \pm 9$ & $67 \pm 5$ \\
\hline SEVO & $68 \pm 7$ & $68 \pm 6$ & $58 \pm 7$ & $64 \pm 5$ & $62 \pm 6$ & $66 \pm 9$ & $64 \pm 8$ \\
\hline ASA & $69 \pm 10$ & $65 \pm 9$ & $61 \pm 4$ & $68 \pm 4$ & $68 \pm 6$ & $67 \pm 7$ & $69 \pm 6$ \\
\hline SEVO+ASA & $73 \pm 11$ & $72 \pm 12$ & $62 \pm 8$ & $60 \pm 8$ & $71 \pm 16$ & $65 \pm 9$ & $71 \pm 17$ \\
\hline SC-560 & $69 \pm 13$ & $69 \pm 12$ & $57 \pm 11$ & $65 \pm 17$ & $63 \pm 11$ & $59 \pm 4$ & $66 \pm 10$ \\
\hline SEVO+SC-560 & $72 \pm 5$ & $69 \pm 6$ & $66 \pm 6$ & $58 \pm 6^{\mathrm{b}}$ & $61 \pm 6$ & $68 \pm 9$ & $62 \pm 7$ \\
\hline NS-398 & $74 \pm 4$ & $75 \pm 4$ & $68 \pm 7$ & $72 \pm 14$ & $70 \pm 13$ & $70 \pm 10$ & $69 \pm 8$ \\
\hline SEVO+NS-398 & $78 \pm 6$ & $76 \pm 7$ & $65 \pm 7^{b}$ & $63 \pm 14$ & $73 \pm 17$ & $72 \pm 6$ & $69 \pm 9$ \\
\hline
\end{tabular}

Abbreviations: ASA, acetylsalicylic acid; BL, baseline; CAO, coronary artery occlusion; CON, control; DMSO, dimethyl sulfoxide; NS-398, a selective cyclooxygenase- 2 inhibitor; SC-560, a selective cyclooxygenase-I inhibitor; SEVO, sevoflurane.

${ }^{2}$ Data are mean $\pm S D$. Data were analyzed at the end of the baseline period; before, during, and after CAO; and 60, I20, and I80 minutes after the onset of reperfusion as indicated by triangles in Figure $\mathrm{I}$.

bSignificantly $(P<.05)$ different from baseline.

post-CAO vs reperfusion for 60 minutes vs reperfusion for 120 minutes vs reperfusion for 180 minutes). In case of any significant main effects or interactions, post hoc 1-way ANOVAs were conducted for each group and each time point. Statistical analysis of data was performed using SPSS 19.0 software (The Apache Software Foundation, Forest Hill, Maryland). Changes in means were considered statistically significant when $P<.05$. Data are presented as mean \pm standard deviation (SD).

\section{Results}

Overall, 68 mice were included in the study to obtain 63 successful experiments. Five animals were excluded because of pump failure during $\mathrm{CAO}$ ( 2 in the SEVO+SC-560 group, 1 in the NS-398 group, 1 in the SEVO+NS-398 group) or because the AAR was less than $20 \%$ (1 in the SEVO+SC-560 group).

\section{Hemodynamic Parameters and AAR}

Hemodynamic parameters at baseline and AAR were not different among groups (Tables 1 and 2 and Figure 2). Compared with baseline values, the heart rate was decreased during the administration of sevoflurane, although statistical significance was only observed in the SEVO and the SEVO+ASA groups. It was not present if sevoflurane was administered in combination with SC-560 or NS-398. Mean arterial pressure (MAP) was significantly decreased during CAO compared with the respective baseline values in the DMSO and SEVO+NS-398 groups. During sevoflurane administration, MAP was significantly decreased in the SEVO+SC-560 group.

\section{Myocardial Infarct Size}

Myocardial infarct size (IS/AAR) was $44 \% \pm 9 \%$ in the control group (Figures 3 and 4). Neither the vehicle dimethyl sulfoxide (DMSO; $42 \% \pm 7 \%$ ) nor the nonselective COX inhibitor acetylsalicylic acid (ASA; 36\% $\pm 6 \%$ ) alone altered the resulting infarct size. Application of 1.0 MAC sevoflurane during early reperfusion significantly reduced the ischemic injury compared with control animals (SEVO; $17 \% \pm 4 \%$ ). The nonselective COX inhibitor ASA did not abolish sevoflurane-induced postconditioning (SEVO+ASA; $16 \% \pm 5 \%$ ). Inhibition of COX-1 using the selective inhibitor SC-560 reduced the IS to $26 \% \pm$ $10 \%$ (SC-560) while not affecting sevoflurane-induced 
Table 2. Body Weight and Planimetry. ${ }^{a}$

\begin{tabular}{|c|c|c|c|c|c|c|c|c|}
\hline & $\mathrm{n}$ & BW, g & $\mathrm{LV}, \mathrm{mg}$ & LV/BW, \% & $\mathrm{AAR}, \mathrm{mg}$ & IS, mg & IS/LV, \% & AAR/LV, \% \\
\hline $\mathrm{CON}$ & 7 & $25.3 \pm 2.0$ & $70.4 \pm 7.9$ & $0.28 \pm 0.02$ & $23.0 \pm 9.7$ & $10.0 \pm 4.5$ & $13.9 \pm 5.0$ & $32.1 \pm 11.2$ \\
\hline DMSO & 7 & $25.3 \pm 3.4$ & $76.1 \pm 7.2$ & $0.30 \pm 0.02$ & $24.5 \pm 8.2$ & $10.2 \pm 3.6$ & $13.3 \pm 4.5$ & $32.3 \pm 11.5$ \\
\hline SEVO & 7 & $25.1 \pm 1.6$ & $69.8 \pm 7.6$ & $0.28 \pm 0.02$ & $23.7 \pm 5.1$ & $3.9 \pm 1.1^{b}$ & $5.6 \pm 1.5^{b}$ & $34.1 \pm 7.3$ \\
\hline ASA & 7 & $25.1 \pm 2.2$ & $72.9 \pm 9.5$ & $0.29 \pm 0.02$ & $22.0 \pm 4.9$ & $7.8 \pm 2.3$ & $10.9 \pm 3.0$ & $30.3 \pm 5.5$ \\
\hline SEVO+ASA & 7 & $25.1 \pm 2.9$ & $68.2 \pm 8.7$ & $0.27 \pm 0.03$ & $21.0 \pm 4.8$ & $3.5 \pm 1.6^{b}$ & $5.1 \pm 1.7^{b}$ & $30.5 \pm 4.5$ \\
\hline SC-560 & 7 & $25.0 \pm 2.2$ & $72.5 \pm 9.2$ & $0.29 \pm 0.04$ & $20.2 \pm 5.0$ & $5.5 \pm 3.1^{b}$ & $7.3 \pm 3.7^{b}$ & $27.6 \pm 3.7$ \\
\hline SEVO+SC-560 & 7 & $27.0 \pm 2.4$ & $77.5 \pm 5.9$ & $0.29 \pm 0.02$ & $27.6 \pm 4.7$ & $6.1 \pm 1.4^{b}$ & $8.0 \pm 2.0^{b}$ & $35.6 \pm 5.4$ \\
\hline NS-398 & 7 & $26.0 \pm 1.9$ & $73.3 \pm 4.4$ & $0.28 \pm 0.02$ & $22.2 \pm 6.1$ & $9.6 \pm 4.6$ & $13.3 \pm 6.6$ & $30.2 \pm 7.5$ \\
\hline SEVO+NS-398 & 7 & $24.6 \pm 2.1$ & $73.5 \pm 5.7$ & $0.30 \pm 0.02$ & $28.7 \pm 9.6$ & $9.3 \pm 4.0$ & $12.7 \pm 5.8$ & $39.4 \pm 14.8$ \\
\hline
\end{tabular}

Abbreviations: AAR, area at risk; ASA, acetylsalicylic acid; BW, body weight; CON, control; DMSO, dimethyl sulfoxide; IS, infarct size; LV, left ventricle; NS-398, a selective cyclooxygenase-2 inhibitor; SC-560, a selective cyclooxygenase-I inhibitor; SEVO, sevoflurane.

${ }^{a}$ Data are mean \pm SD.

${ }^{\mathrm{b}}$ Significantly $(P<.05)$ different from CON.

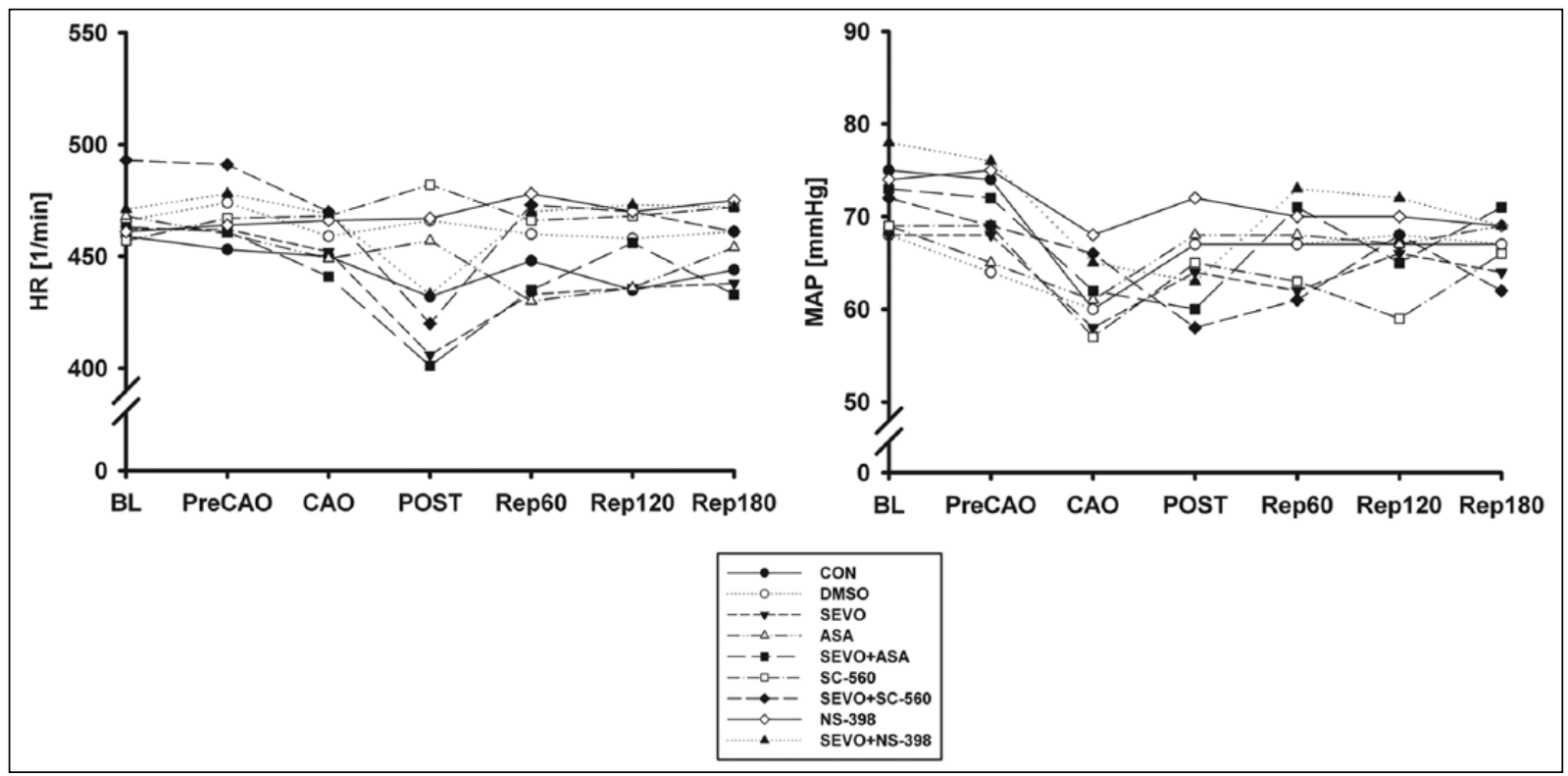

Figure 2. Mean values of heart rate (HR) and mean arterial pressure (MAP). For better visibility, standard deviations and indicators of significance are not shown. Please refer to Table I for further information. Data were analyzed at the end of the baseline period (BL); before (PreCAO), during (CAO), and after (POST) coronary artery occlusion; and 60, 120 , and 180 minutes after the onset of reperfusion (Rep60, Rep 120, and Rep I80). ASA, acetylsalicylic acid; CON, control; DMSO, dimethyl sulfoxide; NS-398, a selective cyclooxygenase-2 inhibitor; SC-560, a selective cyclooxygenase-I inhibitor; SEVO, sevoflurane.

postconditioning (SEVO+SC-560; 22\% $\pm 4 \%$ ). The selective COX-2 inhibitor NS-398 alone did not affect myocardial IS (NS-398; 44\% $\pm 18 \%$ ) but abolished the protective effects provided by sevoflurane-induced postconditioning (SEVO+NS-398; 33\% $\pm 14 \%$ ).

\section{Discussion}

The present study investigates the role of both cyclooxygenase-1 (COX-1) and cyclooxygenase-2 (COX-2) in sevoflurane-induced postconditioning (APOC) against myocardial infarction using a murine in vivo model. The results demonstrate a major role for $\mathrm{COX}-2$ within the signal transduction pathway of APOC, whereas COX-1 is dispensable in this model.

The volatile anesthetic sevoflurane was administered during early reperfusion after coronary artery occlusion and conferred cardioprotection as reflected by a pronounced reduction in myocardial infarct size compared with control animals. Sevoflurane-induced postconditioning (APOC) reduces myocardial infarct size up to $60 \%$ in this mouse model. Cardioprotection by APOC has been described in different species and models, such as in isolated rat hearts, ${ }^{17}$ and in the rat ${ }^{18}$ and rabbit myocardium in 


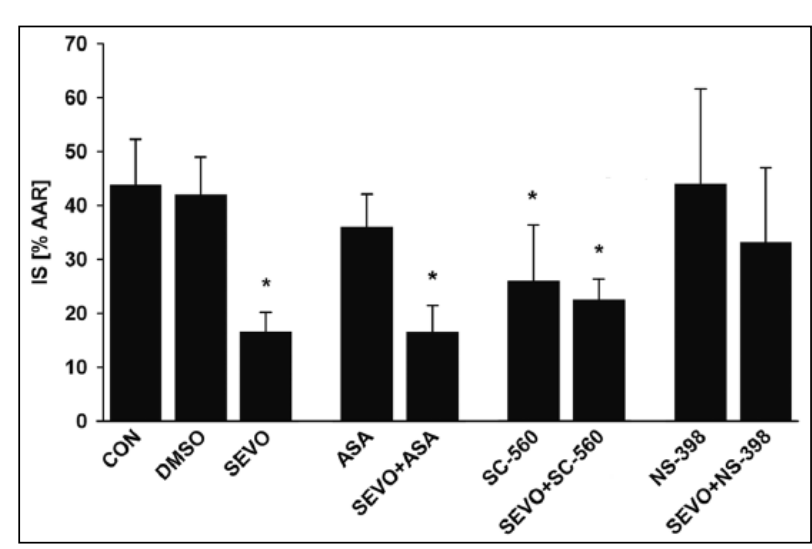

Figure 3. Sevoflurane-induced postconditioning against myocardial infarction is mediated by cyclooxygenase-2 (COX2 ), whereas blockade of cyclooxygenase-I (COX-I) itself is cardioprotective. Myocardial infarct size (IS) expressed as percentage of left ventricular area at risk (AAR). Values are mean \pm SD. $n=7$ in each group. *Significantly $(P>.05)$ different from CON. Mice received either no intervention (CON), dimethyl sulfoxide (DMSO), sevoflurane (SEVO), acetylsalicylic acid alone (ASA) or in combination with sevoflurane (SEVO+ASA), SC-560 alone (SC-560) or in combination with sevoflurane (SEVO+SC-560), or NS-398 alone (NS-398) or in combination with sevoflurane (SEVO+NS-398).

vivo. ${ }^{19}$ Former studies in a murine model reported an infarct size reduction of up to $70 \% .{ }^{20}$ Redel et al ${ }^{20}$ demonstrated that sevoflurane-induced postconditioning was as effective as ischemic postconditioning and sevofluraneinduced pre-conditioning. ${ }^{20}$ The current results confirm the previously reported data regarding the cardioprotective ability of sevoflurane-induced postconditioning in mice.

The results further indicate an important role of COX-2 within the signal transduction cascade of sevofluraneinduced postconditioning. We used NS-398 at a dose that was previously shown to sufficiently block COX-2 in mice. ${ }^{16}$ The isoform COX-2 is known to be an important mediator of early and delayed ischemic ${ }^{1,21}$ and anestheticinduced $^{1,22}$ preconditioning. Additionally, other studies indicate a major role of COX regarding ischemic ${ }^{23}$ and anesthetic-induced ${ }^{24}$ postconditioning. Particularly, COX-2 appears essential regarding reperfusion injury in mammals ${ }^{25}$ and preconditioning by noble gases. ${ }^{26,27}$ The results of the current study are in line with these results, indicating the indispensability of COX-2 in the signaling cascade of sevoflurane-induced postconditioning. In rats, ischemic postconditioning was shown to be COX-2 dependent since the selective COX-2 inhibitor celecoxib abolished the infarct-sparing effect of combining late ischemic preconditioning with postconditioning. ${ }^{28}$ The authors suggested that postconditioning enhances the enzymatic activity of COX-2 induced via late preconditioning. The results of the current study are in line with these results, demonstrating that sevoflurane-induced postconditioning is dependent on COX-2-activity.

Anesthetic-induced postconditioning is a very complex phenomenon that depends on the activation of many intracellular targets. The exact signaling mechanisms are not completely understood. It is known that volatile anesthetics activate several prosurvival kinases such as PI3K/Akt and Erk1/2, which in turn prevents the formation of proapoptotic proteins. ${ }^{29}$ This cascade has been named the reperfusion injury salvage kinase (RISK) pathway. ${ }^{30}$ The protective cascades converge to the mitochondria and reduce the opening probability of the mitochondrial permeability transition pore (mPTP) ${ }^{31}$ This preserves mitochondrial and cellular integrity.

Furthermore, selective inhibition of COX-1 was cardioprotective in this model. Myocardial infarct size was reduced to an extent similar to that achievable with APOC. This finding is remarkable since earlier studies were not able to demonstrate this effect. ${ }^{16,32}$ SC-560 as the COX-1 inhibitor was used at a dose that recently has been demonstrated to effectively block COX-1 in the murine heart during I/R-injury. ${ }^{16}$ Potential unselective intrinsic effects of SC-560 on other components in the signaling cascade thus remain speculative. However, SC-560 is a frequently used inhibitor in rodent reperfusion-injury studies, and other selective COX-1 inhibitors like FR $122047^{33}$ and mofezo$\mathrm{lac}^{34}$ have not been tested. One might further speculate that selective COX-1 inhibition leads to a shift of the thromboxane/prostaglandin balance toward the prostaglandins, which in turn causes vasodilatation of coronary arteries. This might result in increased myocardial oxygen supply during the reperfusion period, thus decreasing the resulting myocardial infarct size. Moreover, the preponderance of prostaglandins might reduce platelet aggregation, ${ }^{35}$ helping to prevent coronary microemboli during reperfusion. Nevertheless, these various effects could have been actuated by any available COX inhibitor due to different affinities and variety of mechanisms.

Whereas COX-2 is a well-known mediator of early ${ }^{1}$ and late ischemic ${ }^{21}$ and anesthetic-induced ${ }^{22}$ preconditioning, the role of COX-1 is not clear regarding anestheticinduced preconditioning. It has been demonstrated that COX-1 mediates the delayed phase (after 48 hours) but not the early phase (after 24 hours) of morphine-induced delayed preconditioning. ${ }^{16}$ Functional blockade of COX-1 by SC-560 neither inhibited APOC nor had any additive beneficial effect on APOC in the current study. Additionally, the beneficial effect of APOC was not altered by pretreatment with acetylsalicylic acid (ASA). Primarily ASA inhibits COX $-1 .^{36}$ These results confirm that interference between anesthetic-induced preconditioning and COX inhibition most likely depends on the COX-2 affinity. However, we found no cardioprotective effect of ASA itself. This emphasizes the relevance of comedications and 


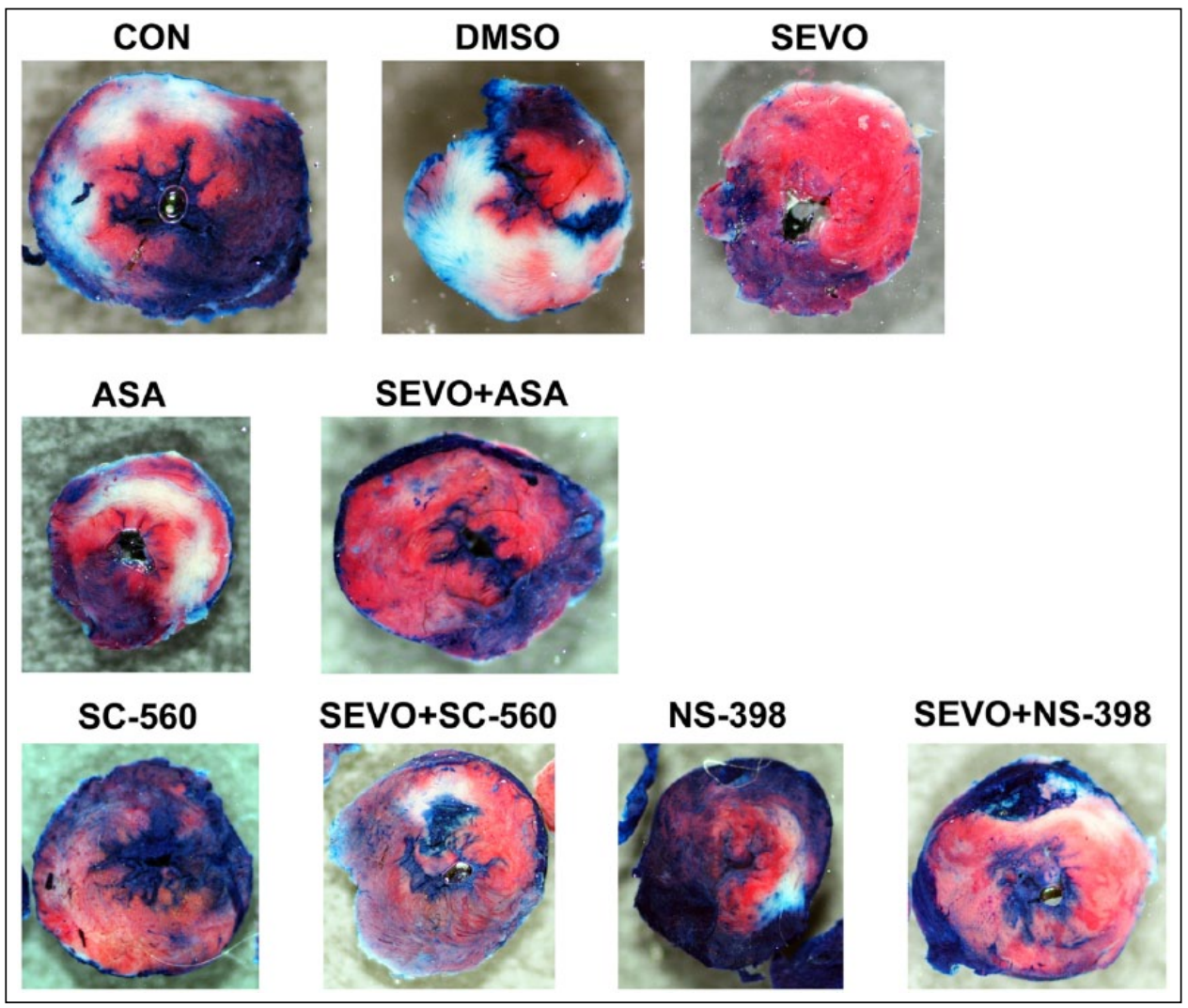

Figure 4. Representative left ventricular slices of an animal of each experimental group. White colored zones indicate infarcted tissue, red colored zones indicate the area at risk (ischemic tissue), and blue colored zones indicate the normal zone (nonischemic tissue). ASA, acetylsalicylic acid; CON, control; DMSO, dimethyl sulfoxide; NS-398, cyclooxygenase-2 blocker; SC-560,

cyclooxygenase-I blocker; SEVO, sevoflurane.

their interference with protective effects subsequent to ischemic injury, a topic relevant for several endangered tissues, such as the kidney ${ }^{37,38}$ and liver. ${ }^{39}$

The results of the current study should be interpreted within the constraint of several potential limitations. The left ventricular area at risk and the amount of coronary collateral blood flow are crucial determinants of myocardial infarct size. However, the area at risk was not different among groups. Coronary collateral blood flow was not measured in this study. However, rodents are reported to have little if any coronary collateral blood flow. ${ }^{40}$ Thus, it is unlikely that AAR and coronary collateral blood flow account for the differences in myocardial infarct size. Furthermore, triphenyltetrazolium chloride (TTC) staining was the only indicator of myocardial infarct size. Other markers of myocardial injury such as troponin values were not determined in this study. Alterations in myocardial oxygen supply and demand ratio might have affected the observed results. Sevoflurane decreased heart rates during early reperfusion in the SEVO and SEVO+ASA groups and tended to decrease heart rates in the SEVO+SC-560 and SEVO+NS-398 groups. Coronary venous oxygen content was not measured, and myocardial oxygen consumption was not directly quantified in this study. Therefore, we cannot completely exclude that changes in myocardial oxygen supply-demand ratio might contribute to the infarct size reduction by sevofluraneinduced postconditioning. It has been shown that SC-560 at $10 \mu \mathrm{g} / \mathrm{g}$ and NS-398 at $5 \mu \mathrm{g} / \mathrm{g}$ effectively inhibit COX-1 and COX-2, respectively. ${ }^{16}$ However, potential effects of SC-560 and NS-398 on other proteins involved in the signal transduction cascade of sevoflurane-induced postconditioning cannot be excluded.

In summary, the present study demonstrates that the in vivo administration of 1.0 MAC sevoflurane during early reperfusion induces postconditioning against myocardial infarction in the murine heart. The results further indicate that these protective effects are mediated by COX-2.

\section{Declaration of Conflicting Interests}

The author(s) declared no potential conflicts of interest with respect to the research, authorship, and/or publication of this article. 


\section{Funding}

The author(s) received no financial support for the research, authorship, and/or publication of this article.

\section{References}

1. Alcindor D, Krolikowski JG, Pagel PS, Warltier DC, Kersten JR. Cyclooxygenase-2 mediates ischemic, anesthetic, and pharmacologic preconditioning in vivo. Anesthesiology. 2004;100:547-554.

2. Lange M, Smul TM, Blomeyer CA, et al. Role of the beta1adrenergic pathway in anesthetic and ischemic preconditioning against myocardial infarction in the rabbit heart in vivo. Anesthesiology. 2006;105:503-510.

3. Smul TM, Lange M, Redel A, Burkhard N, Roewer N, Kehl F. Desflurane-induced preconditioning against myocardial infarction is mediated by nitric oxide. Anesthesiology. 2006;105:719-725.

4. Chiari PC, Bienengraeber MW, Pagel PS, Krolikowski JG, Kersten JR, Warltier DC. Isoflurane protects against myocardial infarction during early reperfusion by activation of phosphatidylinositol-3-kinase signal transduction: evidence for anesthetic-induced postconditioning in rabbits. Anesthesiology. 2005;102:102-109.

5. Deyhimy DI, Fleming NW, Brodkin IG, Liu H. Anesthetic preconditioning combined with postconditioning offers no additional benefit over preconditioning or postconditioning alone. Anesth Analg. 2007;105:316-324.

6. Stumpner J, Smul TM, Redel A, et al. Desflurane-induced and ischaemic postconditioning against myocardial infarction are mediated by Pim-1 kinase. Acta Anaesthesiol Scand. 2012;56:904-913.

7. De Hert SG, Van der Linden PJ, Cromheecke S, et al. Cardioprotective properties of sevoflurane in patients undergoing coronary surgery with cardiopulmonary bypass are related to the modalities of its administration. Anesthesiology. 2004;101:299-310.

8. Bresalier RS, Sandler RS, Quan H, et al. Cardiovascular events associated with rofecoxib in a colorectal adenoma chemoprevention trial. N Engl J Med. 2005;352:1092-1102.

9. Solomon SD, McMurray JJ, Pfeffer MA, et al. Cardiovascular risk associated with celecoxib in a clinical trial for colorectal adenoma prevention. N Engl J Med. 2005;352:1071-1080.

10. Nussmeier NA, Whelton AA, Brown MT, et al. Complications of the COX-2 inhibitors parecoxib and valdecoxib after cardiac surgery. N Engl J Med. 2005;352:1081-1091.

11. Bayne K. Revised Guide for the Care and Use of Laboratory Animals available. American Physiological Society. Physiologist. 1996;39:199, 208-211.

12. Redel A, Jazbutyte V, Smul TM, et al. Impact of ischemia and reperfusion times on myocardial infarct size in mice in vivo. Exp Biol Med (Maywood). 2008;233:84-93.

13. Stumpner J, Redel A, Kellermann A, et al. Differential role of Pim-1 kinase in anesthetic-induced and ischemic preconditioning against myocardial infarction. Anesthesiology. 2009; 111:1257-1264.

14. Eckle T, Grenz A, Kohler D, et al. Systematic evaluation of a novel model for cardiac ischemic preconditioning in mice. Am J Physiol Heart Circ Physiol. 2006;291:H2533-H2540.
15. Liao M, Laster MJ, Eger EI, Tang M, Sonner JM. Naloxone does not increase the minimum alveolar anesthetic concentration of sevoflurane in mice. Anesth Analg. 2006;102:1452-1455.

16. Jiang X, Shi E, Nakajima Y, Sato S, Ohno K, Yue H. Cyclooxygenase-1 mediates the final stage of morphineinduced delayed cardioprotection in concert with cyclooxygenase-2. J Am Coll Cardiol. 2005;45:1707-1715.

17. Chen HT, Yang CX, Li H, et al. Cardioprotection of sevoflurane postconditioning by activating extracellular signalregulated kinase $1 / 2$ in isolated rat hearts. Acta Pharmacol Sin. 2008;29:931-941.

18. Huhn R, Heinen A, Weber NC, Hollmann MW, Schlack W, Preckel B. Hyperglycaemia blocks sevoflurane-induced postconditioning in the rat heart in vivo: cardioprotection can be restored by blocking the mitochondrial permeability transition pore. Br J Anaesth. 2008;100:465-471.

19. Preckel B, Schlack W, Comfere T, Obal D, Barthel H, Thamer V. Effects of enflurane, isoflurane, sevoflurane and desflurane on reperfusion injury after regional myocardial ischaemia in the rabbit heart in vivo. Br J Anaesth. 1998;81:905-912.

20. Redel A, Stumpner J, Tischer-Zeitz T, et al. Comparison of isoflurane-, sevoflurane-, and desflurane-induced pre- and postconditioning against myocardial infarction in mice in vivo. Exp Biol Med (Maywood). 2009;234:1186-1191.

21. Guo Y, Bao W, Wu WJ, Shinmura K, Tang XL, Bolli R. Evidence for an essential role of cyclooxygenase-2 as a mediator of the late phase of ischemic preconditioning in mice. Basic Res Cardiol. 2000;95:479-484.

22. Tanaka K, Ludwig LM, Krolikowski JG, et al. Isoflurane produces delayed preconditioning against myocardial ischemia and reperfusion injury: role of cyclooxygenase- 2 . Anesthesiology. 2004;100:525-531.

23. Penna C, Mancardi D, Tullio F, Pagliaro P. Postconditioning and intermittent bradykinin induced cardioprotection require cyclooxygenase activation and prostacyclin release during reperfusion. Basic Res Cardiol. 2008;103:368-377.

24. Tosaka S, Tosaka R, Matsumoto S, Maekawa T, Cho S, Sumikawa K. Roles of cyclooxygenase 2 in sevofluraneand olprinone-induced early phase of preconditioning and postconditioning against myocardial infarction in rat hearts. $J$ Cardiovasc Pharmacol Ther. 2011;16:72-78.

25. Schmelzle M, Felix SB, Staudt A, Herda LR. Cardioprotection of 17,18-epoxyeicostetraenoic acid in ischemia/reperfusion is mediated by cyclooxygenase-2: a study in a rat model. Transplant Proc. 2011;43:1515-1519.

26. Weber NC, Frassdorf J, Ratajczak C, et al. Xenon induces late cardiac preconditioning in vivo: a role for cyclooxygenase 2? Anesth Analg. 2008;107:1807-1813.

27. Huhn R, Heinen A, Weber NC, et al. Helium-induced late preconditioning in the rat heart in vivo. Br J Anaesth. 2009;102:614-619.

28. Sato H, Bolli R, Rokosh GD, et al. The cardioprotection of the late phase of ischemic preconditioning is enhanced by postconditioning via a COX-2-mediated mechanism in conscious rats. Am J Physiol Heart Circ Physiol. 2007;293:H2557-H2564.

29. Pagel PS. Postconditioning by volatile anesthetics: salvaging ischemic myocardium at reperfusion by activation of prosurvival signaling. J Cardiothorac Vasc Anesth. 2008;22:753-765. 
30. Hausenloy DJ, Yellon DM. New directions for protecting the heart against ischaemia-reperfusion injury: targeting the reperfusion injury salvage kinase (RISK)-pathway. Cardiovasc Res. 2004;61:448-460.

31. Davidson SM, Hausenloy D, Duchen MR, Yellon DM. Signalling via the reperfusion injury signalling kinase (RISK) pathway links closure of the mitochondrial permeability transition pore to cardioprotection. Int J Biochem Cell Biol. 2006;38:414-419.

32. Shinmura K, Nagai M, Tamaki K, Tani M, Bolli R. COX-2derived prostacyclin mediates opioid-induced late phase of preconditioning in isolated rat hearts. Am J Physiol Heart Circ Physiol. 2002;283:H2534-H2543.

33. Abe T, Hayasaka Y, Zhang XY, Hayasaka S. Effects of intravenous administration of FR122047 (a selective cyclooxygenase 1 inhibitor) and FR188582 (a selective cyclooxygenase 2 inhibitor) on prostaglandin-E2-induced aqueous flare elevation in pigmented rabbits. Ophthalmic Res. 2004;36:321-326.

34. Goto K, Ochi H, Yasunaga Y, et al. Analgesic effect of mofezolac, a non-steroidal anti-inflammatory drug, against phenylquinone-induced acute pain in mice. Prostaglandins Other Lipid Mediat. 1998;56:245-254.
35. Dohi M, Sakata Y, Seki J, et al. The anti-platelet actions of FR122047, a novel cyclooxygenase inhibitor. Eur $J$ Pharmacol. 1993;243:179-184.

36. Chandrasekharan NV, Dai H, Roos KL, et al. COX-3, a cyclooxygenase-1 variant inhibited by acetaminophen and other analgesic/antipyretic drugs: cloning, structure, and expression. Proc Natl Acad Sci U S A. 2002;99:13926-13931.

37. Yun Y, Duan WG, Chen P, et al. Down-regulation of cyclooxygenase-2 is involved in ischemic postconditioning protection against renal ischemia reperfusion injury in rats. Transplant Proc. 2009; 41:3585-3589.

38. Feitoza CQ, Goncalves GM, Semedo P, et al. Inhibition of COX 1 and 2 prior to renal ischemia/reperfusion injury decreases the development of fibrosis. Mol Med. 2008;14:724-730.

39. Iwasaki W, Kume M, Kudo K, et al. Changes in the fatty acid composition of the liver with the administration of N-3 polyunsaturated fatty acids and the effects on warm ischemia/ reperfusion injury in the rat liver. Shock. 2010;33:306-314.

40. Maxwell MP, Hearse DJ, Yellon DM. Species variation in the coronary collateral circulation during regional myocardial ischaemia: a critical determinant of the rate of evolution and extent of myocardial infarction. Cardiovasc Res. 1987;21:737-746. 